\title{
Optimal Degree Estimation of the Determinant of a Polynomial Matrix
}

\author{
Dimitris Varsamis $^{1, *}$ and Nicholas Karampetakis ${ }^{2}$ \\ ${ }^{1}$ Department of Informatics \& Communications, Technological Educational Institute of Serres, 62124 Serres, Greece \\ ${ }^{2}$ Department of Mathematics, Aristotle University of Thessaloniki, 54124 Thessaloniki, Greece
}

Received: 16 Apr. 2013, Revised: 17 Aug. 2013, Accepted: 19 Aug. 2013

Published online: 1 Mar. 2014

\begin{abstract}
In this paper we present a recursive formula to find the degree of the determinant of a bivariate polynomial matrix. The proposed algorithm returns the optimal estimation of the degree but has a very large computational cost. The recursive formula can be represented as an assignment problem which is solved with the Hungarian method that has a very small computational cost. The contribution of the proposed formula is to reduce the required interpolation points for the evaluation-interpolation technique. That is, we reduce the evaluations which are computations of determinants or inverse matrices.
\end{abstract}

Keywords: polynomial matrix, determinant, degree, assignment problem

\section{Introduction}

The computation of a) the determinant [1], [2], [3], b) the inverse [4], [5], [6], [7], c) the generalized inverse [8] and d) the Drazin matrix [9] of a bivariate polynomial matrix are some problems in control theory. We can solve numerically the above problems with the aim of the bivariate polynomial interpolation. In particular, we use the technique evaluation-interpolation [10], [11], [12]. According to the evaluation-interpolation technique, first, we must evaluate the values of the determinant or the inverse matrix and in sequence interpolate at the evaluate values. In the above procedure we have the critical problem to define the number of initial values which is the number of interpolation points. The number of initial values must be the minimum because the initial values are determinants of inverse matrices. The number of interpolation points depends on the degree of the interpolating polynomial (determinant or element of inverse matrix) [13], [14], [15]. The optimal degree estimation of the determinant is the aim of this work.

Let a two variable polynomial matrix $A(x, y) \in \mathbb{R}[x, y]^{m \times m}$ where

$$
A(x, y)=\left[\begin{array}{ccc}
a_{1,1}(x, y) & \ldots & a_{1, m}(x, y) \\
\vdots & \ddots & \vdots \\
a_{m, 1}(x, y) & \cdots & a_{m, m}(x, y)
\end{array}\right]
$$

We define the total degree matrix $D \in \mathbb{N}^{m \times m}$ where

$$
D=\left[\begin{array}{ccc}
d_{1,1} & \ldots & d_{1, m} \\
\vdots & \ddots & \vdots \\
d_{m, 1} & \cdots & d_{m, m}
\end{array}\right]
$$

with $d_{i, j}=\operatorname{deg}\left[a_{i, j}(x, y)\right]$ where $\operatorname{deg}[$.$] denotes the total$ degree of polynomial.

Similarly, we define the degree matrix in terms of $x$, $D^{x} \in \mathbb{N}^{m \times m}$ where

$$
D^{x}=\left[\begin{array}{ccc}
d_{1,1}^{x} & \cdots & d_{1, m}^{x} \\
\vdots & \ddots & \vdots \\
d_{m, 1}^{x} & \cdots & d_{m, m}^{x}
\end{array}\right]
$$

with $d_{i, j}^{x}=\operatorname{deg}_{x}\left[a_{i, j}(x, y)\right]$ where $\operatorname{deg}_{x}[$.$] denotes the$ greatest degree in terms of variable $z$ and the degree matrix in terms of $y, D^{y} \in \mathbb{N}^{m \times m}$ where

$$
D^{y}=\left[\begin{array}{ccc}
d_{1,1}^{y} & \cdots & d_{1, m}^{y} \\
\vdots & \ddots & \vdots \\
d_{m, 1}^{y} & \cdots & d_{m, m}^{y}
\end{array}\right]
$$

with $d_{i, j}^{y}=\operatorname{deg}_{y}\left[a_{i, j}(x, y)\right]$ where $\operatorname{deg}_{y}[$.$] denotes the$ greatest degree in terms of variable $y$. For the above

\footnotetext{
*Corresponding author e-mail: dvarsam@teiser.gr
} 
elements hold that

$$
d_{i, j} \leq d_{i, j}^{x}+d_{i, j}^{y} \leq 2 \cdot d_{i, j}
$$

According to [5], [8], [6], [7] the upper bound of degree of the determinant of polynomial matrix $A(x, y)$ is

$$
n_{1}=m \cdot d \text { where } d=\max _{1 \leq i, j \leq m}\left\{d_{i, j}\right\}
$$

In this case we need

$$
N_{1}=\left(\begin{array}{c}
n_{1}+2 \\
n_{1}
\end{array}\right)
$$

interpolation points. Additionally, the upper bound of degree of the determinant of polynomial matrix $A(x, y)$ is $n_{2}$ where

$$
\begin{aligned}
n_{2}=\min \left\{\sum_{i=1}^{m}\left(\max _{1 \leq j \leq m}\left\{\operatorname{deg}\left[a_{i, j}(x, y)\right]\right\}\right),\right. \\
\left.\sum_{j=1}^{m}\left(\max _{1 \leq i \leq m}\left\{\operatorname{deg}\left[a_{i, j}(x, y)\right]\right\}\right)\right\}
\end{aligned}
$$

In this case we need

$$
N_{2}=\left(\begin{array}{c}
n_{2}+2 \\
n_{2}
\end{array}\right)
$$

interpolation points.

With the same way we define

$$
n_{3}^{z}=\left(m \cdot d^{z}\right) \text { where } d^{z}=\max _{1 \leq i, j \leq m}\left\{d_{i, j}^{z}\right\}
$$

with $z \in\{x, y\}$ and

$$
\begin{aligned}
n_{4}^{z}=\min \left\{\sum_{i=1}^{m}\left(\max _{1 \leq j \leq m}\left\{\operatorname{deg}_{z}\left[a_{i, j}(x, y)\right]\right\}\right),\right. \\
\left.\sum_{j=1}^{m}\left(\max _{1 \leq i \leq m}\left\{\operatorname{deg}_{z}\left[a_{i, j}(x, y)\right]\right\}\right)\right\}
\end{aligned}
$$

In these cases we need

$$
N_{3}=\left(n_{3}^{x}+1\right) \cdot\left(n_{3}^{y}+1\right)
$$

and

$$
N_{4}=\left(n_{4}^{x}+1\right) \cdot\left(n_{4}^{y}+1\right)
$$

interpolation points respectively.

Example 1.Let the polynomial matrix

$$
A(x, y)=\left[\begin{array}{ccc}
x^{3}-x^{2} y^{2} & 2 x+3 y & 5 x+2 \\
x^{6}+y^{4}-6 x^{4} y^{3} & x^{2}+y^{2}+2 & x^{3}-2 y \\
x^{3} & y & 4
\end{array}\right]
$$

The polynomial matrix $A(x, y)$ has total degree matrix, degree matrix in terms of $x$ and degree matrix in terms of $y$ respectively,

$$
D=\left[\begin{array}{lll}
4 & 1 & 1 \\
7 & 4 & 3 \\
4 & 1 & 0
\end{array}\right]
$$

and

$$
D^{x}=\left[\begin{array}{lll}
3 & 1 & 1 \\
6 & 2 & 3 \\
2 & 0 & 0
\end{array}\right]
$$

and

$$
D^{y}=\left[\begin{array}{lll}
2 & 1 & 0 \\
4 & 2 & 1 \\
2 & 1 & 0
\end{array}\right]
$$

Then, $d=7, d^{x}=6, d^{y}=4$ and $m=3$ thus

$$
\begin{gathered}
n_{1}=m \cdot d=3 \cdot 7=21 \\
n_{3}^{x}=m \cdot d^{x}=18
\end{gathered}
$$

and

$$
n_{3}^{y}=m \cdot d^{y}=12
$$

Additionally, we have

$$
\begin{gathered}
n_{2}=\min \left\{\sum_{i=1}^{m}\left(\max _{1 \leq j \leq m}\left\{\operatorname{deg}\left[a_{i, j}(x)\right]\right\}\right),\right. \\
\left.\sum_{j=1}^{m}\left(\max _{1 \leq i \leq m}\left\{\operatorname{deg}\left[a_{i, j}(x)\right]\right\}\right)\right\}= \\
=\min \{4+7+4,7+4+3\}=14
\end{gathered}
$$

$$
\begin{gathered}
n_{4}^{x}=\min \left\{\sum_{i=1}^{m}\left(\max _{1 \leq j \leq m}\left\{\operatorname{deg}_{x}\left[a_{i, j}(x, y)\right]\right\}\right),\right. \\
\left.\sum_{j=1}^{m}\left(\max _{1 \leq i \leq m}\left\{\operatorname{deg}_{x}\left[a_{i, j}(x, y)\right]\right\}\right)\right\}= \\
=\min \{3+6+2,6+2+3\}=11
\end{gathered}
$$

and

$$
\begin{aligned}
& n_{4}^{y}=\min \left\{\sum_{i=1}^{m}\left(\max _{1 \leq j \leq m}\left\{\operatorname{deg}_{y}\left[a_{i, j}(x, y)\right]\right\}\right),\right. \\
&\left.\sum_{j=1}^{m}\left(\max _{1 \leq i \leq m}\left\{\operatorname{deg}_{y}\left[a_{i, j}(x, y)\right]\right\}\right)\right\}= \\
&=\min \{2+4+2,4+2+1\}=7
\end{aligned}
$$

The numbers of interpolation points are presented in Table 1. 
Table 1: The number of interpolation points for all cases

\begin{tabular}{llll}
\hline$N_{1}$ & $N_{2}$ & $N_{3}$ & $N_{4}$ \\
\hline 253 & 120 & 247 & 96 \\
\hline
\end{tabular}

\section{Main propose}

In the previous approaches, we compute the upper bounds of the total degree or the degrees in terms of variables $x$ or $y$ of the determinant respectively, as well as the number of points which are required for each case. Then, we will define a recursive formula for the computation of the degree of the determinant. The construction of the recursive formula is based on the corresponding recursive formula for the calculation of the determinant.

Let the polynomials $f(x, y)$ and $g(x, y)$. We know that

$$
\operatorname{deg}[f(x, y) \cdot g(x, y)]=\operatorname{deg}[f(x, y)]+\operatorname{deg}[g(x, y)]
$$

and

$$
\operatorname{deg}[f(x, y)+g(x, y)]=\max \{\operatorname{deg}[f(x, y)], \operatorname{deg}[g(x, y)]\}
$$

Moreover, the calculation of the determinant of the polynomial matrix $A(x, y)$ is based on operations of multiplication and addition between the polynomials. Consequently, the respective operations in degrees of polynomials is the sum of the degrees of polynomials when the polynomials multiplied and the maximum degree of polynomials when the polynomials added.

Therefore, we apply the recursive formula for the calculation of the determinant on matrix $D$ by replacing the multiplication with the operation of addition and the adding with the maximum selection. This returns us the degree of polynomial matrix $A(x, y)$.

The above computational procedure is given by the recursive formula

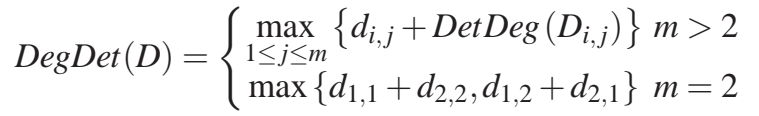

where $D_{i, j} \in \mathbb{N}^{(m-1) \times(m-1)}$ is a sub-matrix of matrix $D$ without the $i$ row and the $j$ column.

Example 2.Let the total degree matrix of the Example 1

$$
D=\left[\begin{array}{lll}
4 & 1 & 1 \\
7 & 4 & 3 \\
4 & 1 & 0
\end{array}\right]
$$

Then

$$
\begin{aligned}
& \operatorname{Deg} \operatorname{Det}(D)=\max \left\{d_{1,1}+\operatorname{Deg} \operatorname{Det}\left(D_{1,1}\right),\right. \\
& \left.d_{1,2}+\operatorname{Deg} \operatorname{Det}\left(D_{1,2}\right), d_{1,3}+\operatorname{Deg} \operatorname{Det}\left(D_{1,3}\right)\right\}
\end{aligned}
$$

where

$$
\begin{aligned}
& \operatorname{Deg} \operatorname{Det}\left(D_{1,1}\right)=\max \left\{d_{2,2}+d_{3,3}, d_{3,2}+d_{2,3}\right\}=4 \\
& \operatorname{Deg} \operatorname{Det}\left(D_{1,2}\right)=\max \left\{d_{2,1}+d_{3,3}, d_{2,3}+d_{3,1}\right\}=7 \\
& \operatorname{Deg} \operatorname{Det}\left(D_{1,3}\right)=\max \left\{d_{2,1}+d_{3,2}, d_{2,2}+d_{3,1}\right\}=8
\end{aligned}
$$

Thus

$$
\operatorname{Deg} \operatorname{Det}(D)=\max \{4+4,1+7,1+8\}=9
$$

instead to $n_{1}=21$ and $n_{2}=14$.

\subsection{Optimal computation of the degree}

The disadvantage of the recursive formula (1) is its computational complexity which is of $O(n !)$. For the computation of DegDet we need $n$ ! combinations of $n$ elements from each column and each row. These elements must be from different rows and different columns. We get the sums $S_{k}$ where $k=1, \ldots, n$ ! of these combinations and we compute the degree

$$
\text { DegDet }=\max _{k=1, \ldots, n !}\left\{S_{k}\right\}
$$

Therefore, this computation can be written as an assignment problem in linear programming. In particular, the objective function is

$$
\max \quad z=\sum_{i=1}^{m} \sum_{j=1}^{m}\left(d_{i, j} \cdot x_{i j}\right)
$$

subject to

$$
\sum_{j=1}^{m} x_{i j}=1 \quad i=1,2, \ldots, m
$$

and

$$
\sum_{i=1}^{m} x_{i j}=1 \quad j=1,2, \ldots, m
$$

with $x_{i j}=\{0,1\}$ for all $i, j$.

The above assignment problem can be solved with the Hungarian method or algorithm [16] which has computational complexity of $O\left(n^{3}\right)$. [17] show that the computational complexity is reduced to $O\left(n^{2} \log n\right)$. The matrix $D$ of the Example 2 with the Hungarian method will be

$$
\left[\begin{array}{lll}
4 & 1 & 1 \\
7 & 4 & 3 \\
4 & 1 & 0
\end{array}\right] \stackrel{\text { Hungarian }}{\text { method }} \longrightarrow\left[\begin{array}{lll}
0 & 0 & 0 \\
0 & 0 & 1 \\
0 & 0 & 1
\end{array}\right]
$$

In the last matrix 2 combinations of 3 independent zeros exists which means the solutions of the problem is the elements $(3,1),(2,2),(1,3)$ or $(2,1),(3,2),(1,3)$ and the objective function is equal to

$$
d_{3,1}+d_{2,2}+d_{1,3}=9
$$

or

$$
d_{2,1}+d_{3,2}+d_{1,3}=9
$$


Table 2: The degree of the determinant and the number of interpolation points for all cases

\begin{tabular}{llllll}
\hline$N_{1}$ & $N_{2}$ & $N_{3}$ & $N_{4}$ & $N_{5}$ & $N_{6}$ \\
\hline 253 & 120 & 247 & 96 & 55 & 48 \\
\hline
\end{tabular}

Example 3.Let the polynomial matrix $A(x, y)$ of the Example1 which has total degree matrix, degree matrix in terms of $x$ and degree matrix in terms of $y$ respectively,

$$
D=\left[\begin{array}{lll}
4 & 1 & 1 \\
7 & 4 & 3 \\
4 & 1 & 0
\end{array}\right] ; D^{x}=\left[\begin{array}{lll}
3 & 1 & 1 \\
6 & 2 & 3 \\
2 & 0 & 0
\end{array}\right] ; D^{y}=\left[\begin{array}{lll}
2 & 1 & 0 \\
4 & 2 & 1 \\
2 & 1 & 0
\end{array}\right]
$$

We have

$$
\begin{aligned}
& n_{5}=\operatorname{Deg} \operatorname{Det}(D)=9 \\
& n_{5}^{x}=\operatorname{Deg} \operatorname{Det}\left(D^{x}\right)=7
\end{aligned}
$$

instead to $n_{3}^{x}=18$ and $n_{4}^{x}=11$ and

$$
n_{5}^{y}=\operatorname{Deg} \operatorname{Det}\left(D^{y}\right)=5
$$

instead to $n_{3}^{y}=12$ and $n_{4}^{y}=7$. The numbers of interpolation points are presented in Table 2.

From the Table 2 we conclude that the reduction of the required interpolation points is significantly large.

\section{Conclusions}

The proposed recursive formula (1) reduces a lot the interpolation points which are required for the calculation of the determinant. This is the advantage of this formula for the technique evaluation-interpolation, because the interpolation points are determinants or adjoint matrices of evaluating matrices which have a very large computational cost. Additionally, the recursive formula (1) is represented as an assignment problem and the computational complexity is of $O\left(n^{2} \log n\right)$. Finally, this formula can be used in one variable polynomial evaluation-interpolation problems such as the computation of the determinant, inverse, Smith form of a polynomial matrix.

\section{Acknowledgements}

The authors wish to acknowledge financial support provided by the Research Committee of the Serres Institute of Education and Technology under grant SAT/IC/28112012-143/4.

\section{References}

[1] L. E. Paccagnella and G. L. Pierobon. Fft calculation of a determinantal polynomial. IEEE Transactions on Automatic Control, 29(3):401-402, 1976.

[2] M Hromcik and Michael Sebek. Numerical and symbolic computation of polynomial matrix determinant. In Proceedings of the 38th IEEE Conference on Decision and Control, volume 2, pages 1887-1888. IEEE, 1999.

[3] Dimitris N. Varsamis and Nicholas P. Karampetakis. On the newton multivariate polynomial interpolation with applications. In 7th International Workshop on Multidimensional (nD) Systems (nDs), 2011, pages 1-8, 2011.

[4] A. Schuster and P. Hippe. Inversion of polynomial matrices by interpolation. IEEE Transactions on Automatic Control, 37:363-365, 1992

[5] S. Vologiannidis and N. P. Karampetakis. Inverses of multivariable polynomial matrices by discrete fourier transforms. Multidimensional Systems and Signal Processing, 15:341-361, 2004.

[6] Nicholas Karampetakis and Alexandros Evripidou. On the computation of the inverse of a two-variable polynomial matrix by interpolation. Multidimensional Systems and Signal Processing, 23:97-118, 2012.

[7] Dimitris N. Varsamis and Nicholas P. Karampetakis. On the Newton bivariate polynomial interpolation with applications. Multidimensional Systems and Signal Processing, 2012. doi:10.1007/s11045-012-0198-z.

[8] Predrag S Stanimirović and Marko D Petković. Computing generalized inverse of polynomial matrices by interpolation. Applied mathematics and computation, 172(1):508-523, 2006.

[9] Marko D Petković and Predrag S Stanimirović. Interpolation algorithm for computing drazin inverse of polynomial matrices. Linear Algebra and its applications, 422(2):526-539, 2007.

[10] A. Bostan and É. Schost. Polynomial evaluation and interpolation on special sets of points. Journal of Complexity, 21(4):420-446, 2005.

[11] J. Kapusta and R. Smarzewski. Fast algorithms for multivariate interpolation and evaluation at special points. Journal of Complexity, 25(4):332-338, 2009.

[12] Dimitris N. Varsamis and Nicholas P. Karampetakis. An optimal bivariate polynomial interpolation basis for the application of the evaluation-interpolation technique. Applied Mathematics \& Information Sciences, 8(1):117125, 2014.

[13] M. Gasca and T. Sauer. Polynomial interpolation in several variables. Advances in Computational Mathematics, 12:377-410, 2000.

[14] P. J. Olver. On multivariate interpolation. Studies in Applied Mathematics, 116:201-240, 2006.

[15] G. M. Phillips. Interpolation and approximation by polynomials. Springer-Verlag, 2003.

[16] Harold W Kuhn. The hungarian method for the assignment problem. Naval research logistics quarterly, 2(1-2):83-97, 1955.

[17] Jack Edmonds and Richard M. Karp. Theoretical improvements in algorithmic efficiency for network flow problems. J. ACM, 19(2):248-264, 1972. 


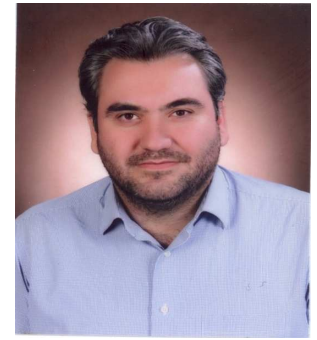

Dimitris
N. Varsamis was born
in Serres, Greece in 1976. He
received the bachelor degree
in Mathematics from the
Department of Mathematics,
University of Ioannina,
Greece and the MSc
in "Theoretical computer
science, control and systems theory" from the Department of Mathematics, Aristotle University of Thessaloniki, Greece. He was awarded the $\mathrm{Ph} . \mathrm{D}$. at the Department of Mathematics (Aristotle University of Thessaloniki, Greece) in 2012, under the supervision of Associate Professor Nicholas Karampetakis. The subject of his doctoral thesis is "On the Development of Computational Methods for the solution of Control Theory problems". From 2001 to 2009 he was a scientific associate with Department of Informatics Communications, Technological Educational Institute of Serres, Greece. In October 2009 he was appointed as Lecturer in Department of Informatics Communications. Dr. Varsamis is the co-author of 20 articles in international journals and conference proceedings.His research interests include Scientific Programming, Computational methods, Control System Theory and Numerical Analysis.

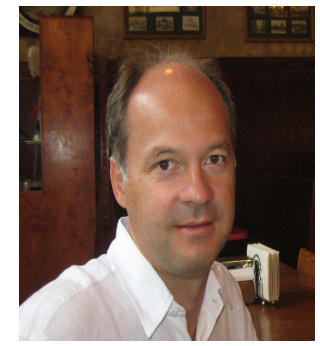

\section{Nicholas}

P. Karampetakis was born in Drama, Greece in 1967. He received the bachelor degree in Mathematics and Ph.D. in Mathematics from the Department of Mathematics, Aristotle University of Thessaloniki, Greece in 1989 and 1993 respectively. From November 1994 to September 1995 he was a research associate with the Department of Mathematical Sciences, Loughborough University of Technology, England. From September 1995 to March 1999 he was a Research Associate with the Department of Mathematics, Aristotle University of Thessaloniki, Greece. From August 2000, to July 2009 he was an Assistant Professor to the same Department, whereas from July 2009, he has been Associate Professor. During the above periods he received many fellowships from the Greek Government, British Council and Engineering and Physical Sciences Research Council of England, and contributes in many research projects sponsored by the Greek Government and the European Union. His present research interests lie mainly in algebraic methods for computer aided design of control systems (CACSD), numerical and symbolic algorithms for CACSD, polynomial matrix theory and issues related to the mathematical systems theory. Dr. Karampetakis is a frequent contributor to the field in the form of journal articles, conference presentations and reviews. He is Senior Member of IEEE and a vice-Chair of the IEEE Action Group on Symbolic Methods for CACSD. He is also an Associate Editor a) of the Journal of Multidimensional Systems and Signal Processing and b) of the International Journal of Applied Mathematics and Computer Science. 